\title{
The Association of Serum Electrolytes with Disease Severity and Obstetric Complications in Pregnant Women with COVID-19: a Prospective Cohort Study from a Tertiary Reference Center
}

\author{
Korrelation zwischen Elektrolytspiegel und Schweregrad \\ der Erkrankung sowie geburtshilflichen Komplikationen \\ bei schwangeren Frauen mit COVID-19: eine prospektive \\ Kohortenstudie in einem Tertiärzentrum
}

\section{(우(1) (요 $\ominus$}

Authors

Atakan Tanacan ${ }^{1}$, Seyit Ahmet Erol ${ }^{1}$, Ali Taner Anuk ${ }^{1}$, Fatma Didem Yucel Yetiskin ${ }^{1}$, Eda Ozden Tokalioglu ${ }^{1}$, Selin Sahin ${ }^{1}$, Serpil Unlu ${ }^{2}$, Huseyin Levent Keskin ${ }^{3}$, Aziz Ahmet Surel ${ }^{4}$, Ozlem Moraloglu Tekin ${ }^{3}$, Dilek Sahin ${ }^{3}$

Affiliations

1 Department of Obstetrics and Gynecology, Turkish Ministry of Health, Ankara City Hospital, Ankara, Turkey

2 Department of Infectious Diseases, Turkish Ministry of Health, Ankara City Hospital, Ankara, Turkey

3 Department of Obstetrics and Gynecology, Turkish Ministry of Health, Ankara City Hospital, University of Health Sciences, Ankara, Turkey

4 Coordinator Head Physician of Turkish Ministry of Health, Ankara City Hospital, Ankara, Turkey

Key words

COVID-19, disease severity, electrolytes, obstetric

complications, pregnancy

Schlüsselwörter

COVID-19, Schweregrad der Erkrankung, Elektrolyten, geburtshilfliche Komplikationen, Schwangerschaft

received 8.6.2021

accepted after revision $\quad 1.8 .2021$

Bibliography

Geburtsh Frauenheilk 2022; 82: 326-332

DOI 10.1055/a-1577-3249

ISSN 0016-5751

(C) 2022. The Author(s).

This is an open access article published by Thieme under the terms of the Creative Commons Attribution-NonDerivative-NonCommercial-License, permitting copying and reproduction so long as the original work is given appropriate credit. Contents may not be used for commercial purposes, or adapted, remixed, transformed or built upon. (https://creativecommons.org/licenses/by-nc-nd/4.0/)

Georg Thieme Verlag KG, Rüdigerstraße 14,

70469 Stuttgart, Germany
Correspondence

Atakan Tanacan

Turkish Ministry of Health Ankara City Hospital,

Department of Obstetrics and Gynecology

Üniversiteler Mahallesi 1604. Cadde No: 9 Çankaya/ANKARA, 06800 Ankara, Turkey

atakantanacan@yahoo.com

ABSTRACT

Introduction To evaluate the association of serum electrolytes with disease severity and obstetric complications in pregnant women with Coronavirus disease 2019 (COVID-19). Materials and Methods This prospective cohort study was conducted on pregnant women with confirmed COVID-19. Study population was divided into two groups: 1) Mild COVID-19 group $(n=811)$ and 2) Moderate/severe COVID-19 group $(n=52)$. Demographic features, clinical characteristics, obstetric complications, and serum electrolytes were compared between the groups. Afterward, a correlation analysis was performed to investigate the association between serum electrolyte disturbances with COVID-19 severity and obstetric complications.

Results Highest serum sodium, hypernatremia, potassium replacement, hypopotassemia, hyperchloremia, initial serum magnesium, hypermagnesemia, and hypocalcemia were significantly higher in the moderate/severe COVID-19 group. The lowest serum sodium, lowest serum potassium, and initial serum calcium were significantly higher in the mild COVID-19 group ( $\mathrm{p}<0.05)$. Statistically significant positive weak correlations were found between hypernatremia, hypopotassemia, hyperchloremia, hypermagnesemia, hypocalcemia and COVID-19 severity ( $r$ values were $0.27,0.20,0.12,0.18$ and 0.12 , $\mathrm{p}$ values were $<0.001,<0.001,0.02,0.03$ and 0.03 , respectively). Furthermore, statistically significant positive weak cor- 
relations were found between hypopotassemia, hypochloremia, hypermagnesemia, and obstetric complications ( $r$ values were $0.10,0.10$, and $0.28, p$ values were $0.004,0.03$, and 0.001 , respectively). A statistically significant negative weak correlation was found between hypomagnesemia and obstetric complications ( $r=-0.23$ and $p=0.01$, respectively).

Conclusion Electrolyte disturbances in pregnant women with COVID-19 seem to be associated with disease severity and obstetric complications.

\section{ZUSAMMENFASSUNG}

Einleitung Ziel der Studie war es, die Verbindung zwischen dem Schweregrad der Erkrankung und den geburtshilflichen Komplikationen bei schwangeren Frauen, die nach Infektion mit SARS-CoV-2 an COVID-19 erkrankt sind, zu evaluieren.

Material und Methoden Diese prospektive Kohortenstudie wurde in einer Gruppe von schwangeren Frauen mit bestätigter COVID-19-Erkrankung durchgeführt. Die Studienpopulation wurde in 2 Gruppen unterteilt: 1) eine Gruppe bestand aus Frauen mit milder COVID-19-Erkrankung ( $n=811)$ und 2 ) eine Gruppe bestand aus Frauen mit mittelschwerer/schwerer COVID-19-Erkrankung $(n=52)$. Die demografischen Merkmale, klinischen Charakteristika, geburtshilflichen Komplikationen und der Elektrolytspiegel beider Gruppen wurden verglichen. Danach wurde eine Korrelationsanalyse durchgeführt, um einen möglichen Zusammenhang zwischen Störungen des Elektrolytspiegels und dem COVID-19-Schweregrad bzw. den geburtshilflichen Komplikationen zu untersuchen.
Ergebnisse Die höchsten Natriumkonzentrationen im Blut, Hypernatriämie, Kaliumsubstitution, Kaliummangel, Hyperchlorämie, die anfänglichen Magnesiumkonzentrationen im Blut, Hypermagnesiämie und Hypokalzämie waren alle signifikant höher in der Gruppe mit mittelschwerer/schwerer COVID-19-Erkrankung. Die tiefsten Natriumkonzentrationen im Blut, tiefsten Kaliumkonzentrationen im Blut und die anfänglichen Kalziumkonzentrationen im Blut waren signifikant höher in der Gruppe mit milder COVID-19-Erkrankung $(p<0,05)$. Es gab statistisch signifikante schwache positive Korrelationen zwischen Hypernatriämie, Hypokaliämie, Hyperchlorämie, Hypermagnesiämie, Hypokalzämie und dem COVID-19-Schweregrad (der r-Wert betrug jeweils 0,27, 0,20, $0,12,0,18$ und 0,12 ; der $p$-Wert betrug jeweils $<0,001$, $<0,001,0,02,0,03$ und 0,03). Es gab auch statistisch signifikante schwache positive Korrelationen zwischen Hypokaliämie, Hyperchlorämie, Hypermagnesiämie und den geburtshilflichen Komplikationen (der r-Wert betrug jeweils 0,10, 0,10 und 0,28; der p-Wert betrug 0,004, 0,03 und 0,001). Eine statistisch signifikante schwache negative Korrelation gab es auch zwischen Hypermagnesiämie und geburtshilflichen Komplikationen $(r=-0,23$ und $p=0,01)$.

Schlussfolgerung Es scheint eine Verbindung zwischen Störungen des Elektrolytspiegels bei schwangeren Frauen, die an COVID-19 erkrankt sind, und dem Schweregrad der Erkrankung bzw. den geburtshilflichen Komplikationen zu geben.

\section{Introduction}

Coronavirus disease 2019 (COVID-19) is an emerging and rapidly evolving health problem. It has caused a global crisis since the beginning of the pandemic and it has forced states to take extraordinary measures [1]. Special attention should be given to pregnant women as pregnancy worsens the clinical course of COVID19 [2,3]. Although most of the cases recover without the need for hospitalization, deterioration of the disease may be rapid in pregnant women [2,3]. Moreover, COVID-19 may cause various obstetric complications like preterm delivery, prelabour rupture of membranes, fetal distress, and increased cesarean delivery rates [2-5]. Although advanced age, presence of comorbidities, genetic predisposition, viral load, and particular laboratory features were reported to be associated with more severe disease, our knowledge is still limited about the course of COVID-19 in pregnant women [6]. On the other hand according to the current literature pregnant women with chronic pre-existing comorbidites like cardiac/pulmonary disease or type 1 diabetes mellitus seem to be affected more seriously $[7,8]$.

Electrolyte imbalance was also reported to be related to severe COVID-19. Hyponatremia, hypopotassemia, hypochloremia, and hypocalcemia were the most common electrolyte abnormalities in the current literature [9-12]. As severe acute respiratory syndrome coronavirus 2 (SARS-CoV-2) mainly attaches to the angio- tensin-converting enzyme 2 (ACE 2) receptors, it may be involved in electrolyte imbalance and impaired electrolyte homeostasis may lead to immune dysregulation [9-12]. Pregnancy is characterized by a variety of physiological changes including increased glomerular filtration rate and renal plasma flow leading to alterations in serum electrolytes $[13,14]$. Thus, the detection, interpretation, and management of electrolyte disturbances during pregnancy may be challenging for physicians. Therefore, the experiences of tertiary reference centers are valuable to establish appropriate management protocols.

The present study aims to evaluate the association of serum electrolytes with disease severity and obstetric complications in pregnant women with COVID-19.

\section{Materials and Methods}

\section{Study design and enrollment of patients}

This prospective cohort study was conducted on pregnant women with confirmed COVID-19 who were followed-up at the Turkish Ministry of Health Ankara City Hospital between 11.03.202030.10.2020. All consecutive cases that gave the required informed consent for participating in the study were included. The study protocol was approved by both the Turkish Ministry of Health and the institutional ethics committee (E2-20-23). 
Turkish Ministry of Health Ankara City Hospital is the largest pandemic center in Turkey and it has played an active role from the beginning of the pandemic. It has served as the main reference center in the region mostly dealing with high-risk pregnancies with approximately 1100 deliveries per month $[15,16]$. COVID-19 was confirmed by SARS-CoV-2 positivity on real-time polymerase chain reaction (RT-PCR) assays of nasopharyngeal and oropharyngeal specimens $[15,16]$. During the study period, only pregnant women with suspected symptoms for COVID-19 were screened in our institution, and universal screening was not performed.

\section{Study groups}

The study population was divided into two groups based on COVID-19 severity:

1. mild COVID-19 group and

2. moderate/severe COVID-19 group.

COVID-19 severity was determined according to current guidelines $[17,18]$.

\section{Study parameters}

Maternal age, pre-pregnancy body mass index (BMI), gravidity, parity, comorbid conditions, gestational age at diagnosis, pregnancy trimester at diagnosis, oxygen support rate, admission to intensive care unit (ICU), radiologic findings consistent with COVID-19 [19], COVID-19 specific medications, antenatal corticosteroid prophylaxis rate, maternal mortality rate, obstetric complications (miscarriage, preterm labor, gestational hypertension, gestational diabetes mellitus, preeclampsia, fetal growth restriction, preterm premature rupture of membranes and intrahepatic cholestasis of pregnancy), and serum electrolyte values were compared between the groups. Obesity was defined as pre-pregnancy $\mathrm{BMI} \geq 30 \mathrm{~kg} / \mathrm{m}^{2}$ [20]. Initial (electrolyte values at admission to hospital), highest, and lowest serum values for sodium, potassium, chloride, magnesium, and calcium were recorded and compared between the groups. Additionally, electrolyte disturbance rates were determined based on pregnancy trimesters and compared between the groups. Corrected calcium values were used for the statistical analysis (measured total calcium [mg/ $\mathrm{dl}]+0.8 \times[4.0$ - serum albumin $\{\mathrm{g} / \mathrm{dl}\}])[13]$. Afterward, a correlation analysis was performed to investigate the association between serum electrolyte disturbances with COVID-19 infection severity and obstetric complications.

\section{Statistical analysis}

Statistical analyses were performed by Statistical Package for the Social Sciences (SPSS 22, IBM SPSS Statistics for Windows, Version 22.0 Armonk, NY: IBM Corp.). Medians and interquartile range values were used for the non-normally distributed descriptive parameters. Mann-Whitney $U$ test was performed for comparing the median values between the groups. Categorical variables were presented with numbers and percentages. $X^{2}$ test was used to compare categorical variables. Correlation analysis was performed by Spearman's rho test. A two-tailed $p$ value $<0.05$ was regarded as statistically significant.

\section{Results}

\section{Main outcomes}

There were 811,40 , and 12 pregnancies with mild, moderate, and severe COVID-19 during the study period. Comparison of demographic features and clinical characteristics between mild and moderate/severe COVID-19 groups was shown in > Table 1. Rates of oxygen support, ICU admission, positive radiologic findings, COVID-19 medication use, antenatal corticosteroid prophylaxis rate, maternal mortality, and obstetric complications were significantly higher in the moderate/severe COVID-19 group $(p<0.05)$.

\section{Comparison of serum electrolytes between the study groups}

Comparison of serum electrolyte values between mild and moderate/severe COVID-19 groups was shown in > Table 2. While serum sodium and potassium values were available for all patients, serum chloride analysis was performed for 365 patients in the mild COVID-19 group and 47 patients in the moderate/severe COVID19 group. Serum magnesium analysis was performed for 115 and 18 patients for mild and moderate/severe COVID-19 infection groups, respectively. Serum calcium analysis was performed for 343 patients in the mild COVID-19 group and 47 patients in the moderate/severe COVID-19 group. The median highest serum sodium was significantly higher in the moderate/severe infection group ( $<0.001)$. The median lowest serum sodium was significantly higher in the mild infection group $(p=0.02)$. Hypernatremia rate was significantly higher in the moderate/severe infection group ( $p<0.001)$. The lowest serum potassium was significantly lower in the moderate/severe infection group $(p<0.001)$. Potassium replacement rate was significantly higher in the moderate/severe infection group $(p<0.001)$. Hypopotassemia rate was significantly higher in the moderate/severe infection group $(p<0.001)$. Hyperchloremia rate and median initial serum magnesium rate were higher in the moderate/severe infection group ( $p$ values were 0.02 and 0.04 , respectively). Hypermagnesemia and hypocalcemia rates were significantly higher in the moderate/severe infection group ( $p=0.03$ for both). Median initial serum calcium was significantly higher in the mild infection group $(p=0.009)$.

\section{Correlation of serum electrolyte disturbances with COVID-19 severity and obstetric complications}

Correlation of serum electrolyte disturbances with COVID-19 severity and obstetric complications was shown in $>$ Table 3. Statistically significant positive weak correlations were found between hypernatremia, hypopotassemia, hyperchloremia, hypermagnesemia, hypocalcemia and COVID-19 severity ( $r$ values were 0.27 , $0.20,0.12,0.18$ and $0.12, p$ values were $<0.001,<0.001,0.02$, 0.03 and 0.03 , respectively). Furthermore, statistically significant positive weak correlations were found between hypopotassemia, hypochloremia, hypermagnesemia, and obstetric complications ( $r$ values were $0.10,0.10$, and $0.28, p$ values were $0.004,0.03$, and 0.001 , respectively). A statistically significant negative weak correlation was found between hypomagnesemia and obstetric complications ( $r=-0.23$ and $p=0.01$, respectively). 
- Table 1 Comparison of demographic features and clinical characteristics between mild COVID-19 group and moderate/severe COVID-19 group.

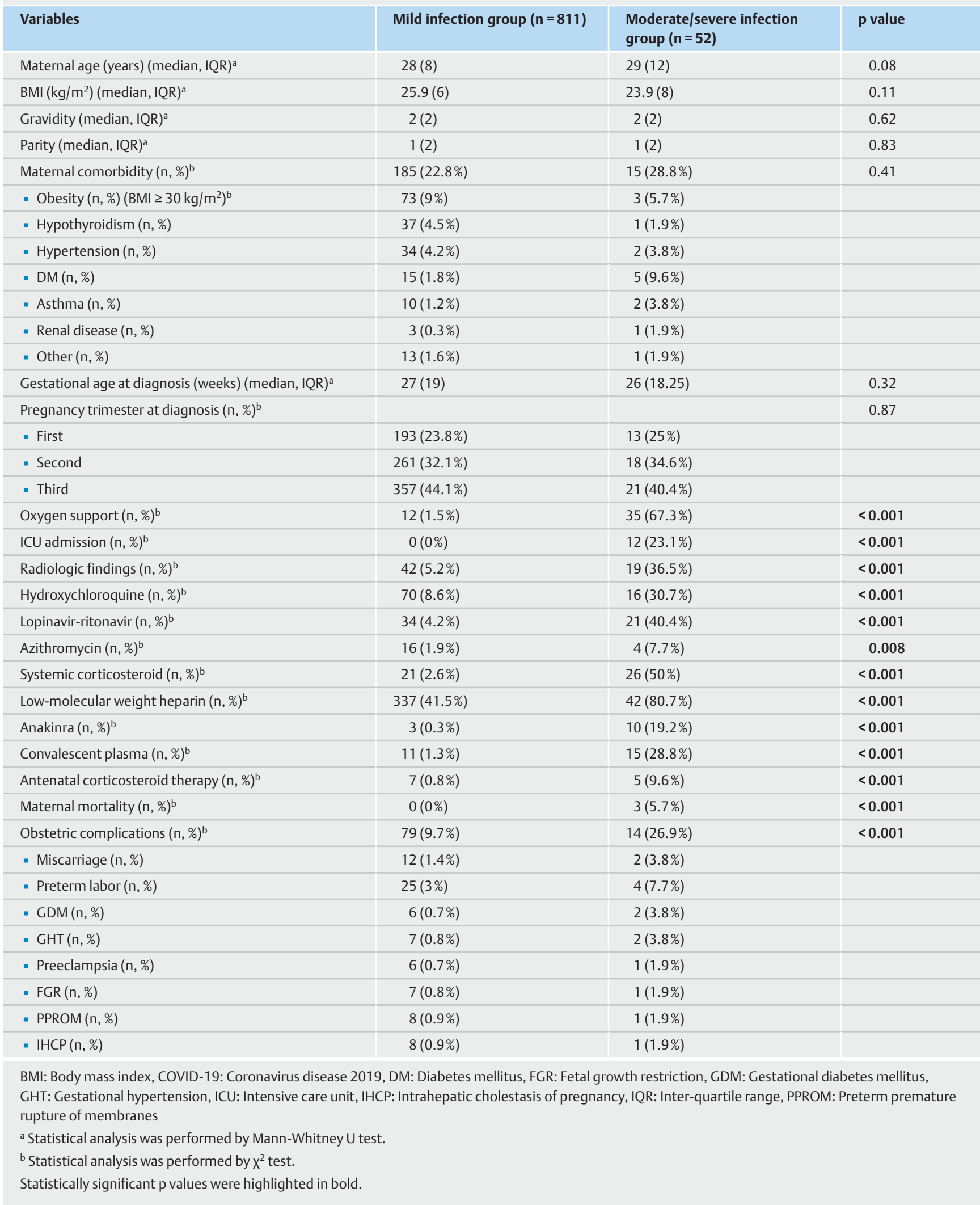


- Table 2 Comparison of serum electrolyte values between mild COVID-19 and moderate/severe COVID-19 group.

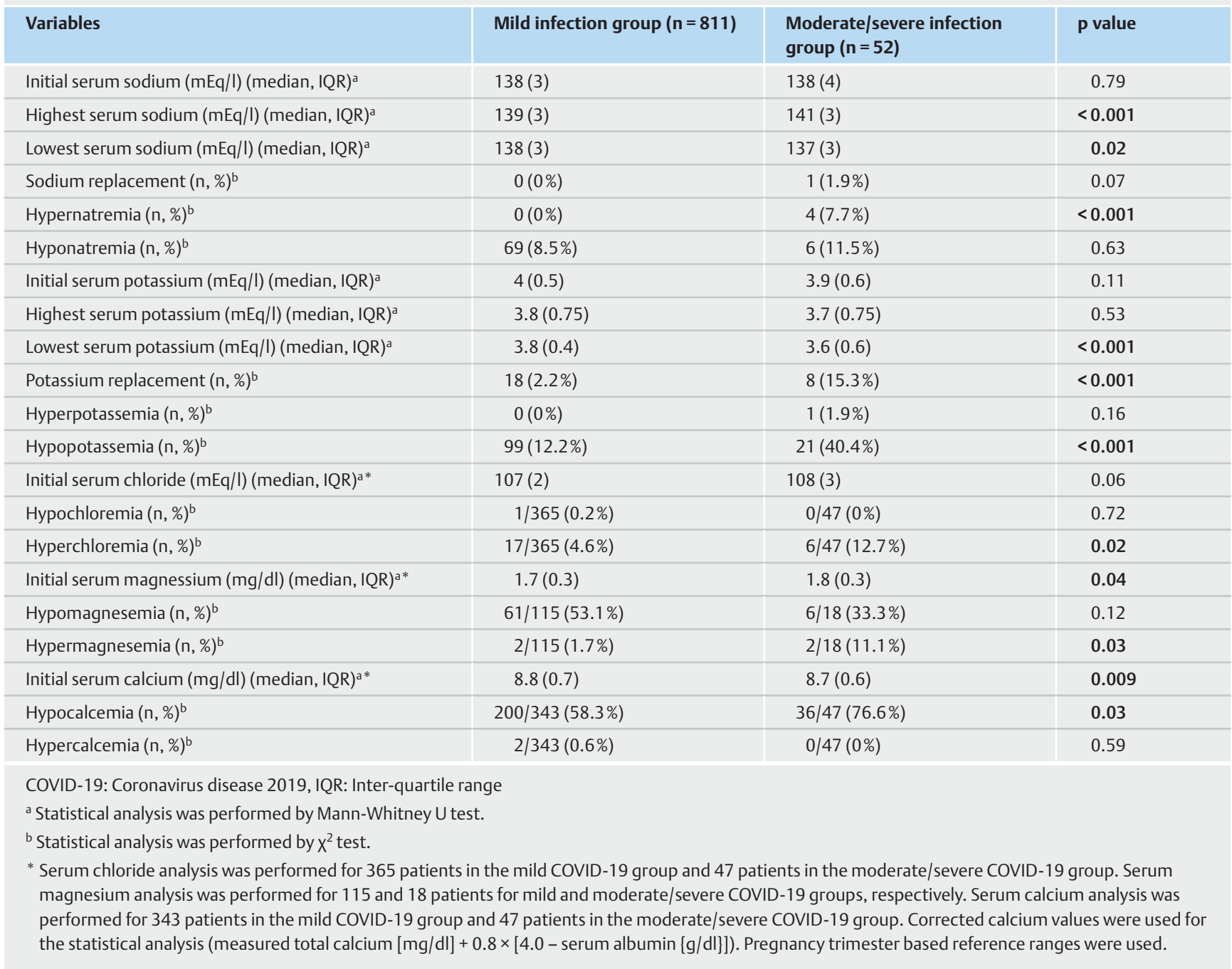

- Table 3 Correlation of serum electrolyte disturbances with COVID-19 severity and obstetric complications.

\begin{tabular}{|c|c|c|c|c|}
\hline \multirow[t]{2}{*}{ Parameter } & \multicolumn{2}{|c|}{ COVID-19 severity } & \multicolumn{2}{|c|}{ Obstetric complications } \\
\hline & r value ${ }^{a}$ & p value ${ }^{a}$ & rvalue ${ }^{a}$ & p value $^{\mathrm{a}}$ \\
\hline Hyponatremia & 0.02 & 0.46 & 0.04 & 0.24 \\
\hline Hypernatremia & 0.27 & $<0.001$ & 0.03 & 0.35 \\
\hline Hypopotassemia & 0.20 & $<0.001$ & 0.10 & 0.004 \\
\hline Hyperpotassemia & 0.27 & 0.17 & 0.25 & 0.21 \\
\hline Hypochloremia & -0.02 & 0.72 & 0.10 & 0.03 \\
\hline Hyperchloremia & 0.12 & 0.02 & -0.03 & 0.56 \\
\hline Hypomagnesemia & -0.13 & 0.12 & -0.23 & 0.01 \\
\hline Hypermagnesemia & 0.18 & 0.03 & 0.28 & 0.001 \\
\hline Hypocalcemia & 0.12 & 0.03 & -0.04 & 0.41 \\
\hline Hypercalcemia & -0.03 & 0.59 & 0.05 & 0.27 \\
\hline
\end{tabular}




\section{Discussion}

The results of the present study indicated that pregnant women with moderate/severe COVID-19 infection had significantly higher electrolyte disturbance rates compared to pregnant women with mild disease. Moreover, altered levels of electrolytes in the study population were associated with COVID-19 severity and obstetric complications.

The major pathophysiological pathways behind the hazardous effect of COVID-19 against the human body have not been completely revealed, yet. Destruction of the respiratory epithelium, fatal arrhythmias, myocardial injury, thromboembolic complications, neuronal involvement, excessive inflammatory response, and predisposition to secondary infections all contribute to disease-related morbidity and mortality $[21,22]$. SARS-CoV-2 enters the host cells via ACE 2 receptors and these receptors are mostly present in respiratory, gastrointestinal, and urinary systems. Binding of SARS-CoV-2 to ACE 2 receptor reduces ACE 2 expression resulting in increased levels of angiotensin 2 [9-12]. As the reninangiotensin-aldosterone system is a major factor in the electrolyte balance, severe COVID-19 may go together with electrolyte disturbances. Furthermore, disease-related nausea, vomiting, and diarrhea may also alter electrolyte balance leading to systemic complications [9-12]. Kidney involvement and inappropriate anti-diuretic hormone syndrome were the other common reasons behind impaired electrolyte balance in infected individuals [912]. Thus, electrolyte disturbances may accompany COVID-19 and physicians should be cautious in the management of these complications. Hyponatremia, hypopotassemia, hypochloremia, and hypocalcemia were the most common complications reported in the literature associated with increased rates of hospitalization, ICU admission, and mortality [9-12]. Serum electrolytes have various functions in the host immunity like activation of lymphocytes, modulation of cytokines, immunoglobulin synthesis, and cell to cell interactions. However, it is unclear whether these electrolyte disturbances lead to disease progression or COVID-19 itself leads to impaired electrolyte imbalance [9-12]. The situation is even more complicated in pregnant patients as serum electrolyte levels are affected by pregnancy-related adaptive changes [13,23]. Generalized vasodilation, increased arterial compliance, decreased systemic vascular resistance, increased cardiac output, including increased renal perfusion and glomerular filtration rate all affect serum levels of electrolytes in pregnant women depending on pregnancy trimesters [24]. For this reason, physicians should be more attentive in the management of electrolyte disturbances in pregnant women with COVID-19.

Hyponatremia was the most common electrolyte imbalance reported in the literature and the majority of the cases were associated with the syndrome of inappropriate antidiuresis [9-12]. Lower levels of serum sodium were observed in patients with pneumonia and sodium deficiency was found to be associated with more severe COVID-19 [9-12]. Both altered renin-angiotensin-aldosterone system and induction of excessive vasopressin release by interleukin 6 are thought to take part in the pathogenesis of lower sodium levels in infected cases [9-12]. However, hyponatremia and sodium replacement rates were similar between the groups in the present study. Yet, extreme values were more prominent in the moderate/severe infection group and hypernatremia rate was significantly higher in the mentioned group. Contrary to the literature only hypernatremia was found to be positively correlated with disease severity [9-12].

Increased rates of urinary potassium extraction were shown in patients with COVID-19 [9-12]. Hypopotassemia was a common condition in infected individuals especially in cases with severe COVID-19. Low potassium level was more significant in patients admitted to ICU and hypopotassemia was considered as a risk factor for cardiac complications [9-12]. Consistent with the literature both hypopotassemia and potassium replacement rates were higher in moderate/severe cases and hypopotassemia was found to be positively correlated with disease severity and obstetric complications in the present study.

Hypochloremia was reported to be associated with unfavorable outcomes in patients with COVID-19 [9-12]. Proximal tubule injury, renal hypoxia, impaired coagulation system, and altered renin-angiotensin-aldosterone system are thought to play a role in the disturbances of serum chloride in infected patients [9-12]. However, differently from the literature hyperchloremia was more frequent in pregnancies with severe infection and hyperchloremia was positively correlated with COVID-19 severity in the present study. On the other hand, hypochloremia was found to be positively correlated with obstetric complications.

Magnesium was reported to take part in vital processes like immune cell adherence, antibody-dependent cytolysis, and immunoglobulin M lymphocyte binding [9-12]. Decreased levels of magnesium in infected cases are thought to be involved in devastating events like cytokine storm and supplementation of magnesium is considered as protective against severe COVID-19 [9-12, 25]. However, contrary to the literature initial serum magnesium and hypermagnesemia rates were significantly higher in cases with moderate/severe COVID-19. Hypermagnesemia was found to be positively correlated with both disease severity and obstetric complications. Furthermore, a negative correlation was observed between hypomagnesemia and obstetric complications.

Calcium was shown to take part in the entrance of SARS-CoV-2 into the host cell [9-12]. Moreover, hypocalcemia was reported to be related to increased rates of ICU admission, need for mechanical ventilation, and mortality [9-12]. Similar to the literature lower serum calcium levels and higher hypocalcemia rates were observed in patients with moderate/severe disease. Additionally, hypocalcemia was found to be positively correlated with disease severity.

Physicians should keep in mind that serum electrolyte levels in pregnant women with COVID-19 may be affected by various factors like maternal physiologic adaptive changes, medications, disease severity, and pregnancy trimesters. Thus, further studies on larger populations with more study parameters are necessary to reach more conclusive results. On the other hand, considering the limited knowledge in the pregnant population, the present study may enlighten the development of future studies focusing on the establishment of more appropriate management protocols and alternative treatment options. To the best of our knowledge, this is the most comprehensive study evaluating the association of serum electrolytes with disease severity and obstetric complications in pregnant women with COVID-19. 
The main strengths of the present study were the large number of cases, the high number of study parameters, and its unique design. On the other hand, lack of information related to the long term outcomes was the main limitation.

\section{Conclusion}

In conclusion, electrolyte disturbances in pregnant women with COVID-19 seem to be associated with disease severity and obstetric complications.

\section{Funding}

No funding was used for this study.

\section{Acknowledgements}

Special thanks to all the health-care professionals in our country who work devotedly for the community at the pandemic period.

\section{Conflict of Interest}

The authors declare that they have no conflict of interest.

\section{References}

[1] Fenwick M, McCahery JA, Vermeulen EPM. Will the World Ever Be the Same After COVID-19? Two Lessons from the First Global Crisis of a Digital Age. Eur Bus Org Law Rev 2021; 22: 125-145

[2] Zambrano LD, Ellington S, Strid P et al.; CDC COVID-19 Response Pregnancy and Infant Linked Outcomes Team. Update: Characteristics of Symptomatic Women of Reproductive Age with Laboratory-Confirmed SARS-CoV-2 Infection by Pregnancy Status - United States, January 22October 3, 2020. MMWR Morb Mortal Wkly Rep 2020; 69: 1641-1647

[3] Allotey J, Stallings E, Bonet M et al.; for PregCOV-19 Living Systematic Review Consortium. Clinical manifestations, risk factors, and maternal and perinatal outcomes of coronavirus disease 2019 in pregnancy: living systematic review and meta-analysis. BMJ 2020; 370: m3320

[4] Bein B, Bachmann M, Huggett S et al. SARS-CoV-2/COVID-19: EvidenceBased Recommendations on Diagnosis and Therapy. Geburtshilfe Frauenheilkd 2020; 80: 491-498

[5] Singh S, Nair VG, Singh VV et al. Pregnancy-Specific Concerns and Psychological Impact of COVID-19 on Antenatal Women. GORM 2021; 1-6 [online ahead of print]

[6] Jordan RE, Adab P, Cheng KK. Covid-19: risk factors for severe disease and death. BMJ 2020; 368: $\mathrm{m} 1198$

[7] Klaritsch P, Ciresa-König A, Pristauz-Telsnigg G; board of the OEGGG. COVID-19 During Pregnancy and Puerperium - A Review by the Austrian Society of Gynaecology and Obstetrics (OEGGG). Geburtshilfe Frauenheilkd 2020; 80: 813-819

[8] Stumpfe FM, Titzmann A, Schneider MO et al. SARS-CoV-2 Infection in Pregnancy - a Review of the Current Literature and Possible Impact on Maternal and Neonatal Outcome. Geburtshilfe Frauenheilkd 2020; 80: 380-390
[9] Lippi G, South AM, Henry BM. Electrolyte imbalances in patients with severe coronavirus disease 2019 (COVID-19). Ann Clin Biochem 2020; 57: 262-265

[10] Tezcan ME, Dogan Gokce G, Sen N et al. Baseline electrolyte abnormalities would be related to poor prognosis in hospitalized coronavirus disease 2019 patients. New Microbes New Infect 2020; 37: 100753

[11] Sarvazad H, Cahngaripour SH, Eskandari Roozbahani N et al. Evaluation of electrolyte status of sodium, potassium and magnesium, and fasting blood sugar at the initial admission of individuals with COVID-19 without underlying disease in Golestan Hospital, Kermanshah. New Microbes New Infect 2020; 38: 100807

[12] Taheri M, Bahrami A, Habibi P et al. A Review on the Serum Electrolytes and Trace Elements Role in the Pathophysiology of COVID-19. Biol Trace Elem Res 2021; 199: 2475-2481

[13] Abbassi-Ghanavati M, Greer LG, Cunningham FG. Pregnancy and laboratory studies: a reference table for clinicians. Obstet Gynecol 2009; 114: 1326-1331

[14] Turğal M, Yazıcıoğlu A, Beksaç K et al. Pregnancy Outcome of Renal Transplant Recipients: Analysis of Nine Patients. GORM 2016; 19: 162164

[15] Sahin D, Tanacan A, Erol SA et al. Updated experience of a tertiary pandemic center on 533 pregnant women with COVID-19 infection: A prospective cohort study from Turkey. Int I Gynaecol Obstet 2021; 152: 328-334

[16] Tanacan A, Erol SA, Turgay B et al. The rate of SARS-CoV-2 positivity in asymptomatic pregnant women admitted to hospital for delivery: Experience of a pandemic center in Turkey. Eur | Obstet Gynecol Reprod Biol 2020; 253: 31-34

[17] Turkish Ministry of Health, General Directorate of Public Health. COViD19 (SARS-CoV-2 infection) Guideline, Scientific Committee Report. Accessed May 01, 2021 at: https://covid19bilgi.saglik.gov.tr/depo/ rehberler/COVID-19_Rehberi.pdf?type=file

[18] Rasmussen SA, Smulian JC, Lednicky JA et al. Coronavirus Disease 2019 (COVID-19) and pregnancy: what obstetricians need to know. Am J Obstet Gynecol 2020; 222: 415-426

[19] Recker F, Weber E, Strizek B et al. Lung Sonography in Obstetrics during COVID-19. Geburtshilfe Frauenheilkd 2020; 80: 1026-1032

[20] Yurtcu E, Mutlu S, Ozkaya E. The Effects of Pre-Pregnancy Body Mass Index and Weight Gain During Pregnancy on Perinatal Outcomes: A Retrospective Cohort Study. GORM 2021 doi:10.21613/GORM.2021.1149

[21] Wiersinga W], Rhodes A, Cheng AC et al. Pathophysiology, Transmission, Diagnosis, and Treatment of Coronavirus Disease 2019 (COVID-19): A Review. JAMA 2020; 324: 782-793

[22] Cavalcante MB, Cavalcante CTMB, Braga ACS et al. COVID-19 Treatment: Drug Safety Prior to Conception and During Pregnancy and Breastfeeding. Geburtshilfe Frauenheilkd 2021; 81: 46-60

[23] Karasu Y, Dilbaz B, Ülker K. Renal Failure in Pregnancy: Case Reports and Review of the Literature. GORM 2016; 21: 161-166

[24] Kohlhepp LM, Hollerich G, Vo L et al. [Physiological changes during pregnancy]. Anaesthesist 2018; 67: 383-396

[25] Anuk AT, Polat N, Akdas S et al. The Relation Between Trace Element Status (Zinc, Copper, Magnesium) and Clinical Outcomes in COVID-19 Infection During Pregnancy. Biol Trace Elem Res 2020. doi:10.1007| s12011-020-02496-y 